\title{
BATTERY CHARGING USING THERMOELECTRIC GENERATION MODULE IN AUTOMOBILES
}

\author{
Adhithya $\mathbf{K}^{\mathbf{1}}$, Rajeshwar Anand ${ }^{2}$, Balaji $\mathbf{G}^{\mathbf{3}}$, Harinarayanan $\mathbf{J}^{4}$ \\ ${ }^{l}$ Bachelor of Engineering, Electrical and Electronics Engineering Dept, Sri Sairam Engineering College, Chennai, \\ India \\ ${ }^{2}$ Bachelor of Engineering, Electrical and Electronics Engineering Dept, Sri Sairam Engineering College, Chennai, \\ India \\ ${ }^{3}$ Bachelor of Engineering, Electrical and Electronics Engineering Dept, Sri Sairam Engineering College, Chennai, \\ India \\ ${ }^{4}$ Assistant Professor Grade II, Electrical and Electronics Engineering Dept, Sri Sairam Engineering College, \\ Chennai, India
}

\begin{abstract}
The idea of this project is to utilise the waste heat energy being generated in automobiles. It involves the trapping of heat energy being generated from the heat source in vehicles and convert to electrical energy which can be used for many appliances. The heat energy and the temperature from the heat source is being sensed by the thermocouple and is converted to electrical energy by a device called Thermoelectric Generator which works on Seebeck effect. The electric potential produced in thermoelectric generator is boosted by the boost converter thereby increasing the magnitude of voltage, required for charging battery. Further, the battery is connected to run the auxiliary appliances in the system.
\end{abstract}

Keywords: Thermocouple; Thermoelectric Generator; Seebeck effect, Boost converter $* * *$

\section{INTRODUCTION}

In the modern world, the electricity is very immportant for our day to day activities inorder to provide comfort to the human beings. Right from simple appliances to complex systems, the electrical energy plays a vital role for the process and operation of these systems. In India, the total installed capacity for the electricity sector is $258.701 \mathrm{GW}$ by January 2015 and the per capita total electricity consumption is $917.2 \mathrm{kWh}$. The renewable energy power plants constitute $28.43 \%$ of total installed capacity and nonrenewable energy power plants constitute $71.57 \%$ of total installed capacity.

In the modern technologies, the production of power is focussing on the renewable energy resources as the nonrenewable energy resources are getting depleted. There are some technologies which implement the recycling of waste energy, by which the waste energy produced at the exhaust can be trapped and recycled into useful form [1] [5].

Every day, the heat energy is produced from its sources is wasted, which includes the hot combustion gases, hot products obtained in the industrial processes and heat transfer from hot equipment surfaces. It includes all kinds of human activities, natural systems and all organisms. The heat energy getting wasted can be trapped and converted to useful electrical energy by the device called thermo-electric generator [1] [2].
The emf produced from the thermoelectric generator module (TEG module) may not be sufficient to supplement enough power to the system. Hence, the power must be steeped up by the use of converters, which include the boost converter [4]. These converters boost the input voltage produced, thereby sufficient for charging the batteries [6]. The battery once charged, can be used for operating the appliances.

\section{HEAT ENERGY}

The heat is a form of energy that is used in our day to day life. This heat energy can be seen in gas stoves at homes, engines in automobiles, compressors and other various equipments. In the heat energy, when there is a suitable physical pathway, the heat flows from the hot surface to the cold surface. It results in a net increase in entropy. The pathway of heat can be direct, as in conduction and radiation, or indirect, as in convection. Hence, the heat refers to the process of transfer, not to the property of a system. The quantity of heat is measured in terms of Joules (J) in SI units. This quantity can be measured by calorimetry, or can be determined by the calculations based on other quantities, which rely on the first law of thermodynamics.

There is an emission of heat due to various processes undergoing in the system. However the magnitude of heat generated at various sources is different; thereby the temperature of these operating equipments is not the same. The transfer of heat energy involves three different types, which include conduction, convection and radiation. This 
heat energy transfer is based upon the devices that emit or work on heat energy. All devices under the heat energy follow the laws of thermodynamics. Some of the devices emitting heat energy include heat engine and heat pump. The heat engine is a device used to run the vehicle by heat energy generated. The waste heat energy is produced at the exhaust. These devices work on the heat energy cycles, depending upon the system.

\section{THERMOELECTRIC POWER}

The Thermoelectric power is the power obtained by the conversion of the generated heat energy into electrical energy. It works on the principle of Seebeck effect, which can be stated as, "When two ends of the conductor are held at different temperatures, the electrons at the hot junction at higher thermal velocities diffuse into the cold junction." [2]. This defines that the thermal electrons move from the junction in the hot region to the junction in the cold region. Therefore, the emf produced in the thermoelectric module is proportional to the temperature difference. The potential difference can be obtained as $\mathrm{V}=\alpha \Delta \mathrm{T}$, where, temperature difference $\Delta \mathrm{T}=\mathrm{T}_{\mathrm{h}}-\mathrm{T}_{\mathrm{c}}$ and $\alpha$ is the Seebeck coefficient or thermo-power expressed in $\mu \mathrm{V}$. The sign of $\alpha$ is positive if emf tends to drive an electric current through the wire from the hot to cold junction and is negative vice versa [2].

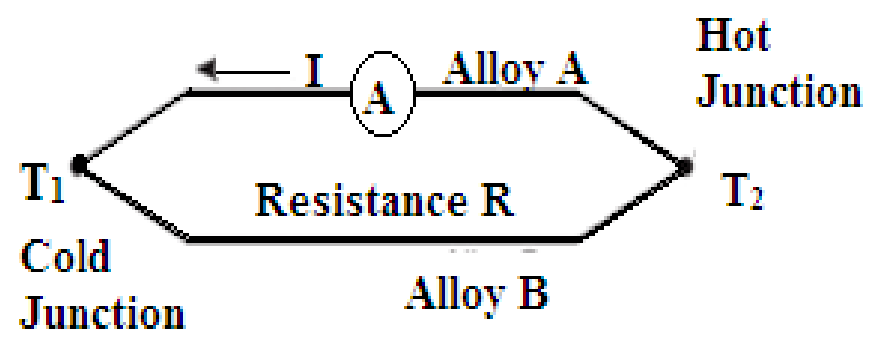

\section{THERMOELECTRIC GENERATOR}

The Thermoelectric Generator, also known as TEG, is a device that works on the principle of Seebeck effect, which is stated above. The TEG module consists of two semiconductor materials, also known as the Seebeck cells or thermo elements [2]. This module has semiconductor thermo elements that are connected electrically in series in order to elevate the resulting voltage and due to the temperature difference between the walls of the plate; the energy that is captured from thermally excited electrons. A single thermocouple comprises of two thermo elements namely $\mathrm{p}$ type and n-type elements. The themo-elements of the n-type and p-type semiconductors are connected thermally in parallel and electrically in series [7].

The TEG module can be used for the generation of electric power, in case of dearth of power. The efficiency of the thermo element depends on the value of load resistance and the property of the semiconducting material. Most of the TEG modules are made up of Bismuth Telluride $\left(\mathrm{Bi}_{2} \mathrm{Te}_{3}\right)$ semiconductor. The heat flowing from one face of the module involves three effects: heat associated with Seebeck effect, the half of Joule heating and thermal conduction of the semiconductor materials. The heterogeneous material composition inside the thermo elements and the dissimilar geometry introduces dissimilarity in both p-type and n-type materials. Hence the analysis of dissimilar elements is considered during calculation. The electric circuit defining the Seebeck coefficient of the p-type and n-type is given as $\alpha=\alpha_{p}-\alpha_{n}$, where $\alpha_{p}$ and $\alpha_{n}$ are the Seebeck coefficients of $p$ type and $n$ type material respectively.

$$
\mathrm{I}=\left(\mathrm{T}_{2}-\mathrm{T}_{1}\right) \mathbf{x}\left(\mathrm{K}_{\mathrm{A}}-\mathrm{K}_{\mathrm{B}}\right) / \mathrm{R}
$$

Fig 1: Seebeck effect

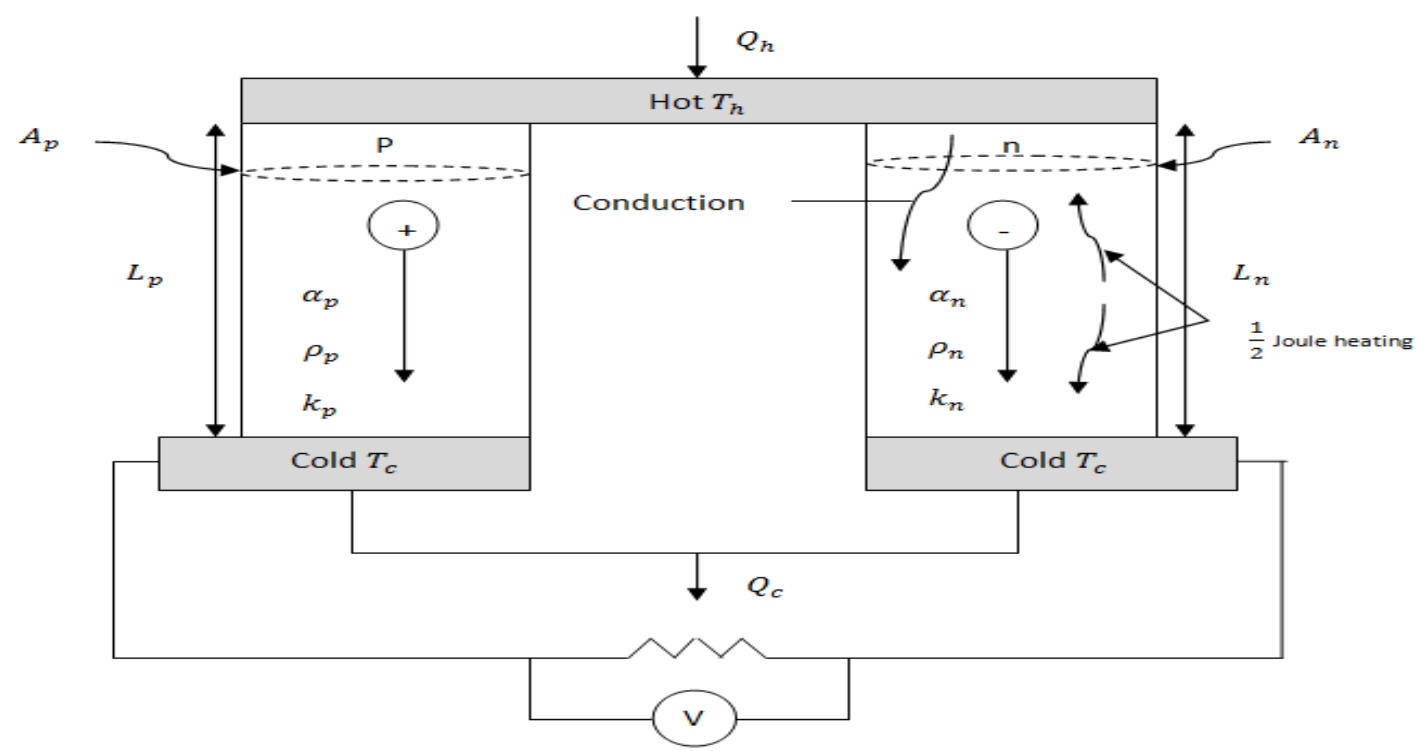

Fig 2: Single thermocouple model 


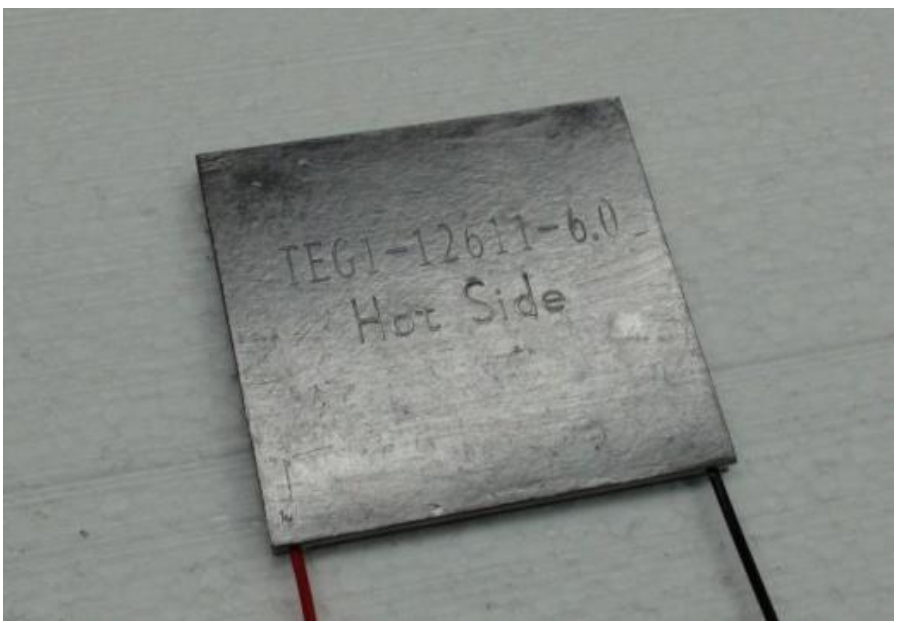

\section{EXPERIMENTAL SETUP}

The experimental setup of this project involves TEG modules placed at the heat source. The output from this module is given as the input to the boost converter, boosting the voltage from TEG. This boosted voltage is a variable DC input voltage used to charge the battery. For the operation of the boost converter, the PWM pulses must be provided to the MOSFET (switching device), which is provided by the MOSFET driver circuit. The whole process in this experiment is controlled by using PIC microcontroller. This circuit can be used in simple applications where heat sources are present [3]. By this method, the waste heat produced at the source can be used for producing electricity [5]. The block diagram for this experiment is as shown below.

Fig 3: Thermoelectric generator

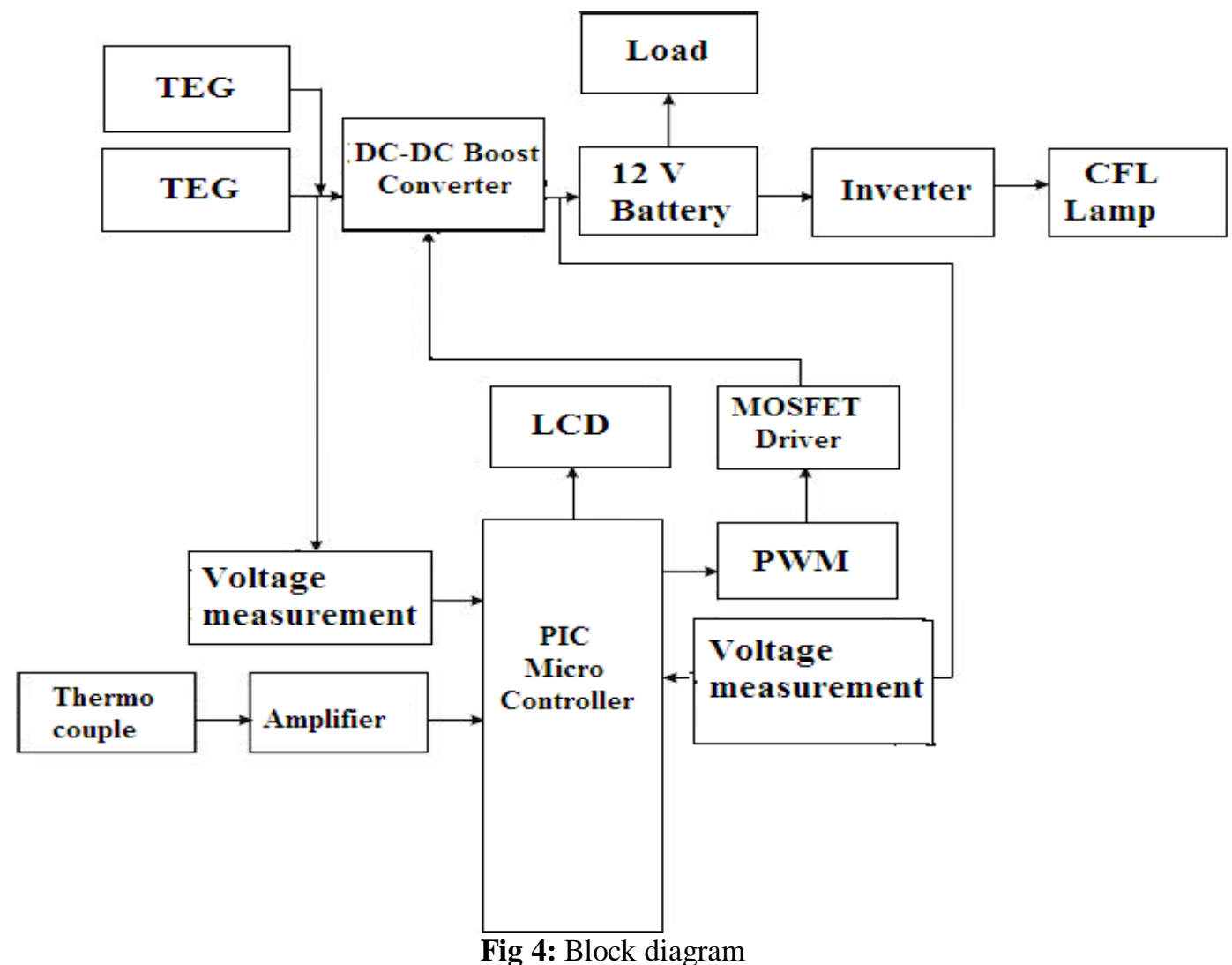

\subsection{Thermoelectric Module}

The thermoelectric module is the thermoelectric generator, which is used to convert the heat energy into electrical energy. They absorb the heat energy present in the source, and by this heat energy, the electrons at the hot junction gets excited. The excited electrons move to the cold junction. With the mothion of electrons from the hot junction to the cold junction, the emf is produced. This project works on the principle of Seebeck effect [1]. Normal thermoelectric modules used are made up of Bismuth Telluride $\left(\mathrm{Bi}_{2} \mathrm{Te}_{3}\right)$ and sometimes, made up of Ceramic Plates (CP). The performance specifications of a nominal TEG is as shown, which depicts the working of modules at different temperatures, their currents, voltages, etc.
Table 1: Parameters of the Module at Different Temperatures

\begin{tabular}{|l|l|l|}
\hline Hot side temperature $\left({ }^{\circ} \mathrm{C}\right)$ & $25^{\circ} \mathrm{C}$ & $50^{\circ} \mathrm{C}$ \\
\hline $\mathrm{Q}_{\max }($ Watts $)$ & 3.5 & 3.9 \\
\hline$\Delta \mathrm{T}_{\max }\left({ }^{\circ} \mathrm{C}\right)$ & 67 & 75 \\
\hline $\mathrm{I}_{\max }(\mathrm{A})$ & 3.0 & 3.0 \\
\hline $\mathrm{V}_{\max }(\mathrm{V})$ & 1.9 & 2.2 \\
\hline Module Resistance $(\Omega)$ & 0.58 & 0.66 \\
\hline
\end{tabular}


The circuit diagram for this module is as shown.

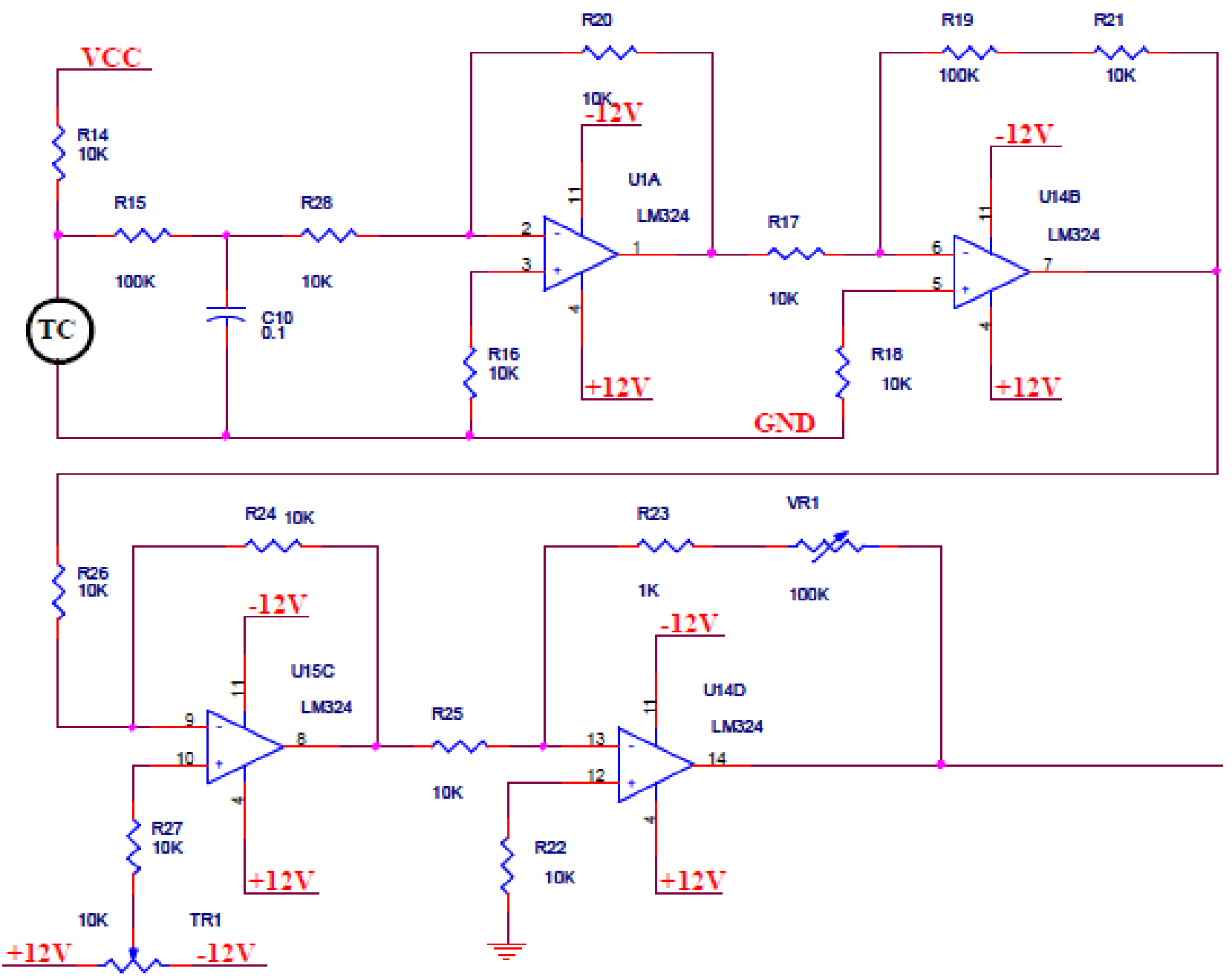

Fig 5: Thermoelectric module circuit diagram

\subsection{Boost Converter}

The boost converter is a device that is used to boost the magnitude of the DC input voltage. It converts the fixed input DC voltage into variable DC voltage. The boost converters use the switched inductors [4], with a switching device which can be the MOSFET. The metal-oxidesemiconductor field-effect transistor (MOSFET, MOS-FET, or MOS FET), is by far the most common field-effect transistor in both digital and analog circuits. The MOSFET is composed of a channel of n-type or p-type semiconductor material and is accordingly called an NMOSFET or a PMOSFET. It is a class of switching-mode power supply (SMPS) containing at least two semiconductor switches (a diode and a transistor) and at least one energy storage element. Filters made of capacitors (sometimes in combination with inductors) are normally added to the output of the converter to reduce output voltage ripple. This boost converter is also called step-up converter as the magnitude of the output voltage is greater than that of input voltage. Since the power $(\mathrm{P}=\mathrm{VI})$ must be conserved, the magnitude of output current is lesser than the source current. The boost converter block used in this circuit is as shown.

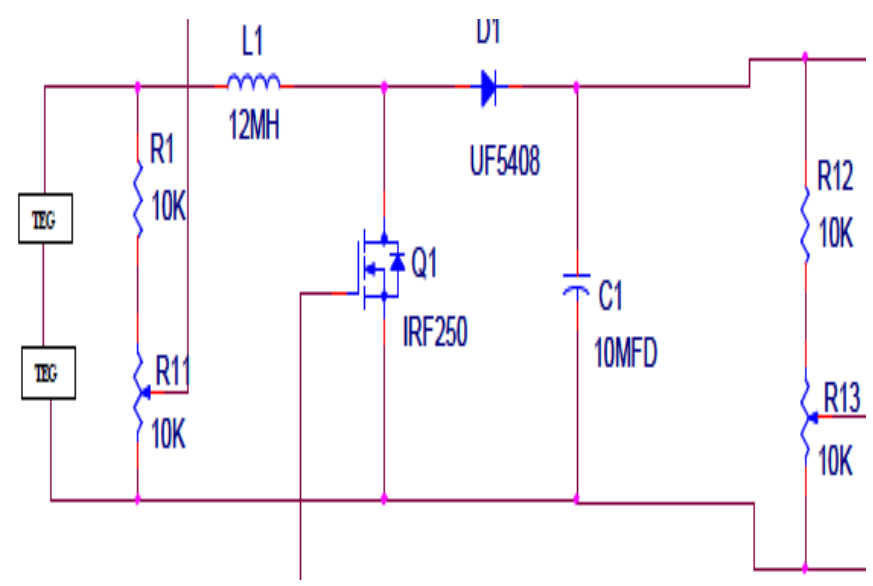

Fig 6: Boost Converter circuit

\subsection{MOSFET Driver Circuit}

The MOSFET driver circuit is the circuit used to control the switching of the MOSFET in the boost converter. This operation of the MOSFET driver circuit is done by the PWM signals. The Pulse Width Modulation (PWM) signals provide high speed switching of the MOSFET. The waves 
are generated by the PIC microcontroller. The PWM time period and duty cycle is controlled by the software. High frequency PWM power control systems are easily realisable with semiconductor switches. The discrete on/off states of the modulation are used to control the state of the switch (es) which correspondingly control the voltage across or current through the load. The major advantage of this system is the switches are either off and not conducting any current, or on and have (ideally) no voltage drop across them. The circuit diagram of MOSFET driver used in this experiment is as shown.

\subsection{PIC Microcontroller}

The PIC microcontroller also called the Peripheral Interface Controller, is a controlling device for the whole circuit process. It plays a major role in providing the PWM pulses to the MOSFET driver circuit. The commonly used PIC microcontroller is PIC 16F877. The core features of PIC microcontroller includes high performance RISC CPU, eight level deep hardware stack, low power consumption (around $2 \mathrm{~mA}$, for $5 \mathrm{~V}, 4 \mathrm{MHz}$ ), wide operating voltage range. It is a 40 pin device, with 5 ports. Port A uses 6 pins, ports $\mathrm{B}, \mathrm{C}$ and $\mathrm{D}$ uses 8 pins and Port $\mathrm{E}$ has 3 pins. The port $\mathrm{D}$ is 8 bit with Schmitt triggered input buffers. The ports $\mathrm{B}$ and $\mathrm{C}$ are 8-bit bi-directional ports. The 3 pins of Port $\mathrm{E}$ are individually configurable as inputs or outputs, these pins also have Schmitt triggered input buffers. The buffers are Schmitt trigger inputs, when they are configured as external interrupts, when used in serial programming modes, when configured as general purpose inputs and TTL input when operated in Parallel Slave Port Mode. Some pins in the microcontroller are multiplexed with alternate function for the peripheral features of the device. The feature of the PIC microcontroller is as shown below.

\begin{tabular}{|l|l|l|l|}
\hline Device & $\begin{array}{l}\text { Program } \\
\text { Flash }\end{array}$ & $\begin{array}{l}\text { DATA } \\
\text { memory }\end{array}$ & $\begin{array}{l}\text { DATA } \\
\text { EEPROM }\end{array}$ \\
\hline $\begin{array}{l}\text { PIC } \\
16 F 877\end{array}$ & $8 \mathrm{~K}$ & 368 Bytes & 256 Bytes \\
\hline
\end{tabular}

The wiring diagram of this microcontroller is explained as shown: The pins 2 and 3 are connected to the boost converter resistances $\mathrm{R} 11$ and $\mathrm{R} 13$. The input from the thermocouple is provided at the pin 4 . The pin 1 is provided as the input to the microcontroller, and the pins 11 and 32 are connected to $5 \mathrm{~V}$ input source, correspondingly pins 31 and 12 are grounded. The pins 13 and 14 are wired to the crystal oscillator of $4 \mathrm{MHz}$; pin 40 provides the PWM signals to the MOSFET driver circuit. The pins 19, 20, 21 , $22,27,28,29$ and 30 are connected to the $16 \times 2$ LCD Display. The diagram for microcontroller is as shown below:

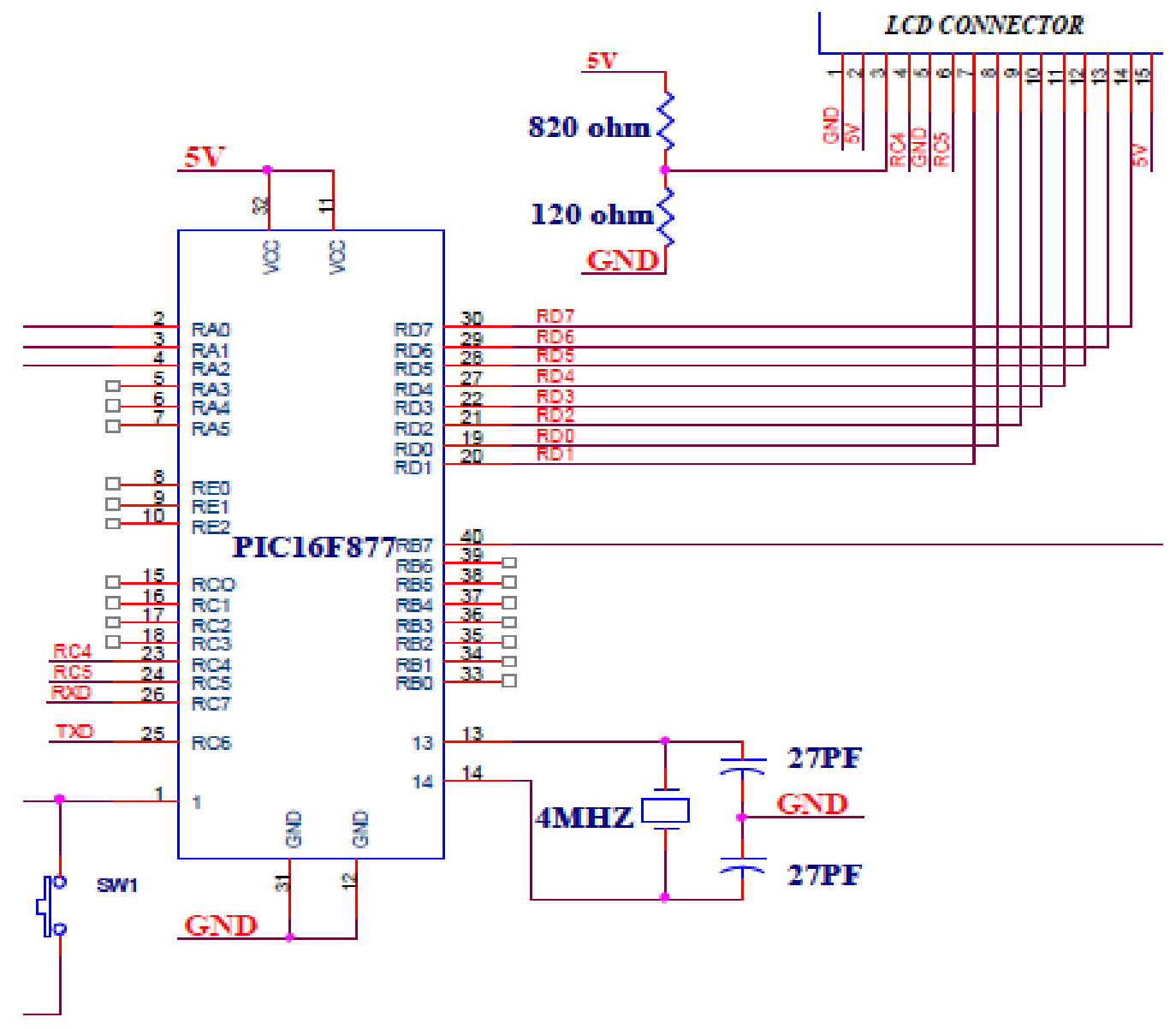

Fig 7: Microcontroller and its wiring 
The other devices used in this circuit include the voltage measuring device, which is used to measure the voltage produced at the TEG module, and the voltage at the boost converter; the Amplifier for amplifying input voltage from the TEG module; thermocouple for measuring the input temperature of the heat source and the LCD display for displaying the parameters in the circuit.

\section{WORKING}

The heat from the heat source in automobiles is monitored and measured by a device called thermocouple. This heat from the source is converted to electrical energy of a smaller magnitude ranging from $2 \mathrm{~V}$ to $3 \mathrm{~V}$. This voltage is amplified by using a voltage amplifier, to around $5 \mathrm{~V}$. This input voltage from the amplifier is boosted by using a boost converter. The boost converter consists of switched inductor, with MOSFET as a switching device. The PWM signals are provided from the PIC microcontroller, which is used to operate the MOSFET driver circuit. The MOSFET driver circuit provides high speed switching for the MOSFET in the boost converter. Based upon the switching, the energy is stored in the inductor and is delivered to the battery. A battery of the order of $12 \mathrm{~V}$ is connected across the boost converter. The variable DC input voltage from the boost converter is used to charge the battery. After the battery is charged, is is used to operate the load. The load circuit in this experiment involves the CFL lamps and a fan, which is operated form this single $12 \mathrm{~V}$ battery. The performance of the circuit depends on the magnitude of heat at the source.

\section{RESULTS}

The magnitude of the input voltage from the TEG module depends upon the temperature of the heat source. The performance curves of the module at $\mathrm{Th}=50^{\circ} \mathrm{C}$ is as shown below.

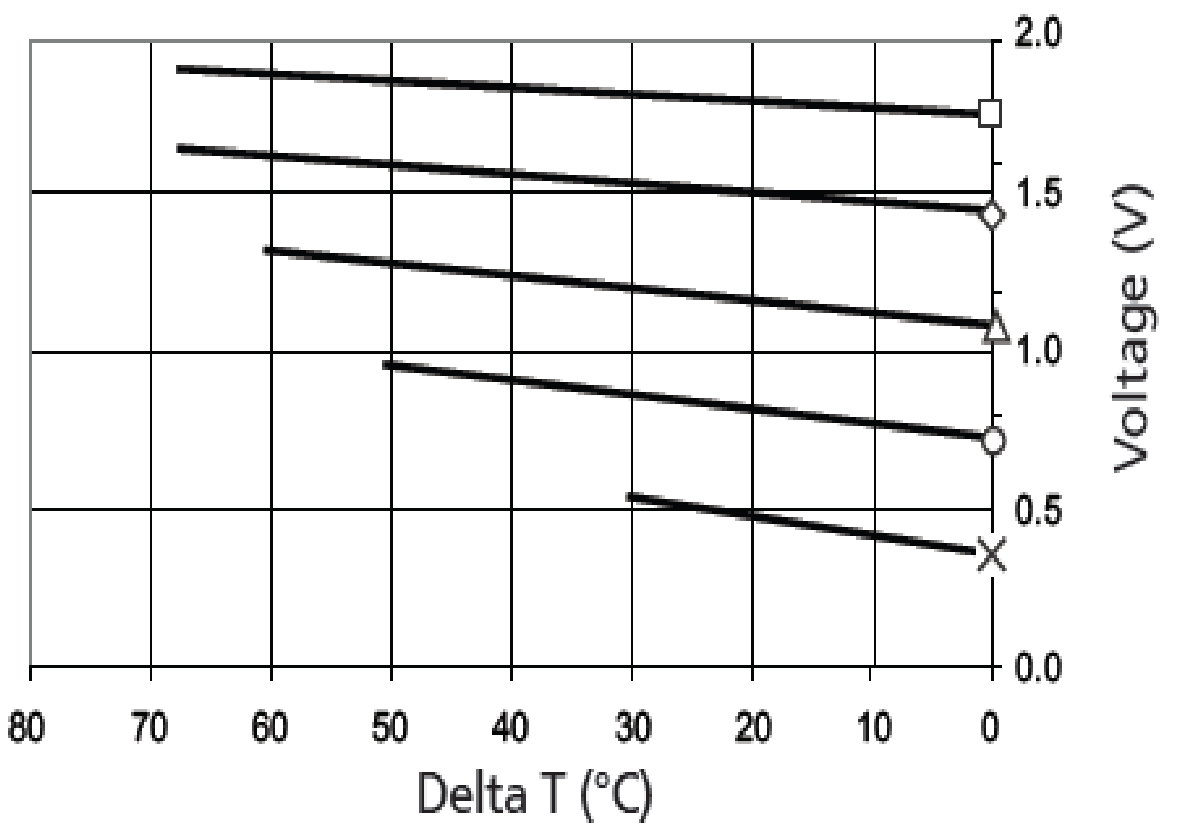

Fig 8: Performance curve at $\mathrm{Th}=50^{\circ} \mathrm{C}$

The input voltage increases with an increase in the temperature difference of the source. The graph is plotted, with temperature difference versus voltage, as shown. 


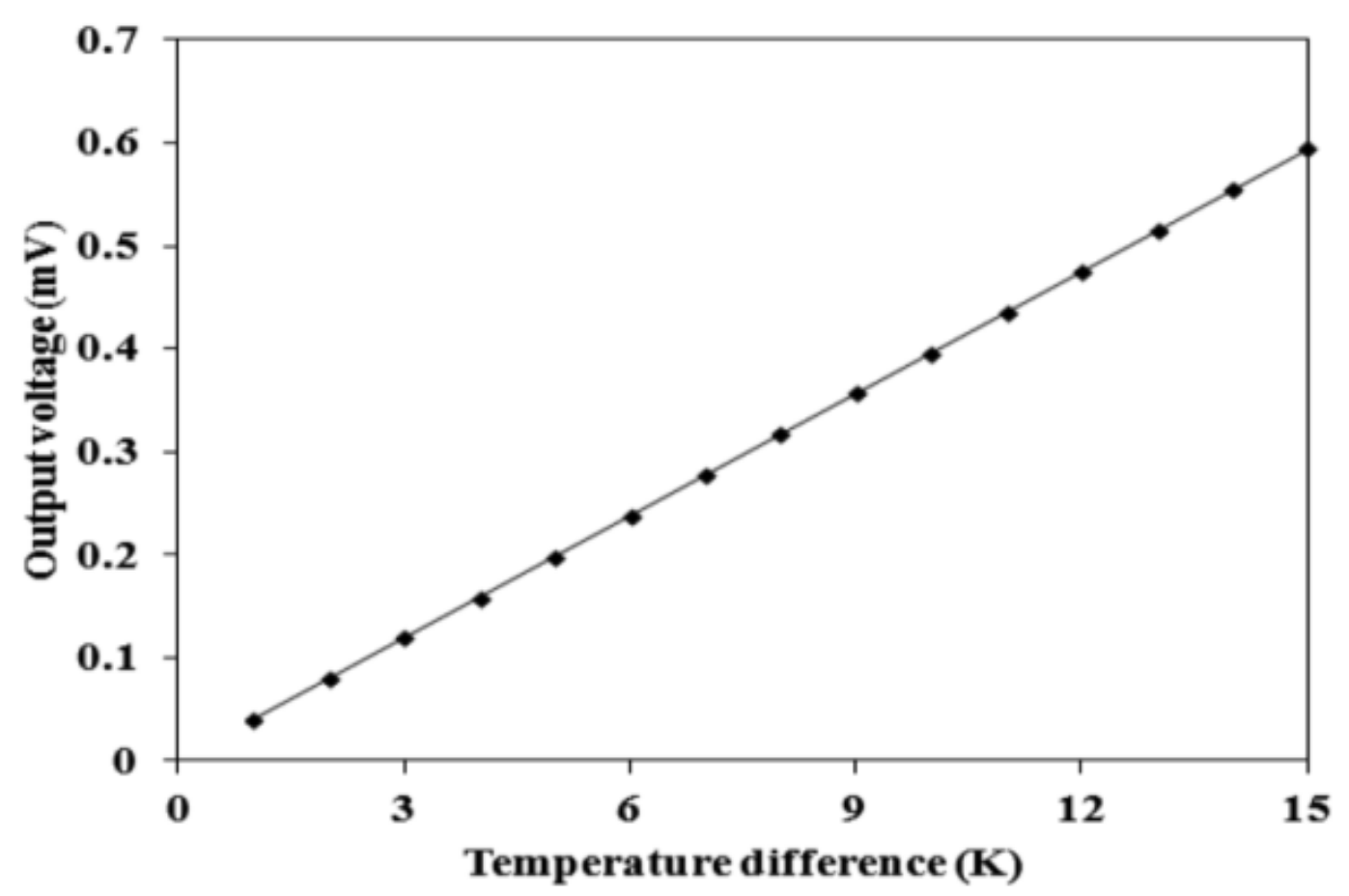

Fig 9: Temperaturre difference vs Voltage graph

The voltage is amplified by the amplifier, this amplified voltage is boosted to a magnitude of higher voltage, and this depends upon the speed of MOSFET switching [4].

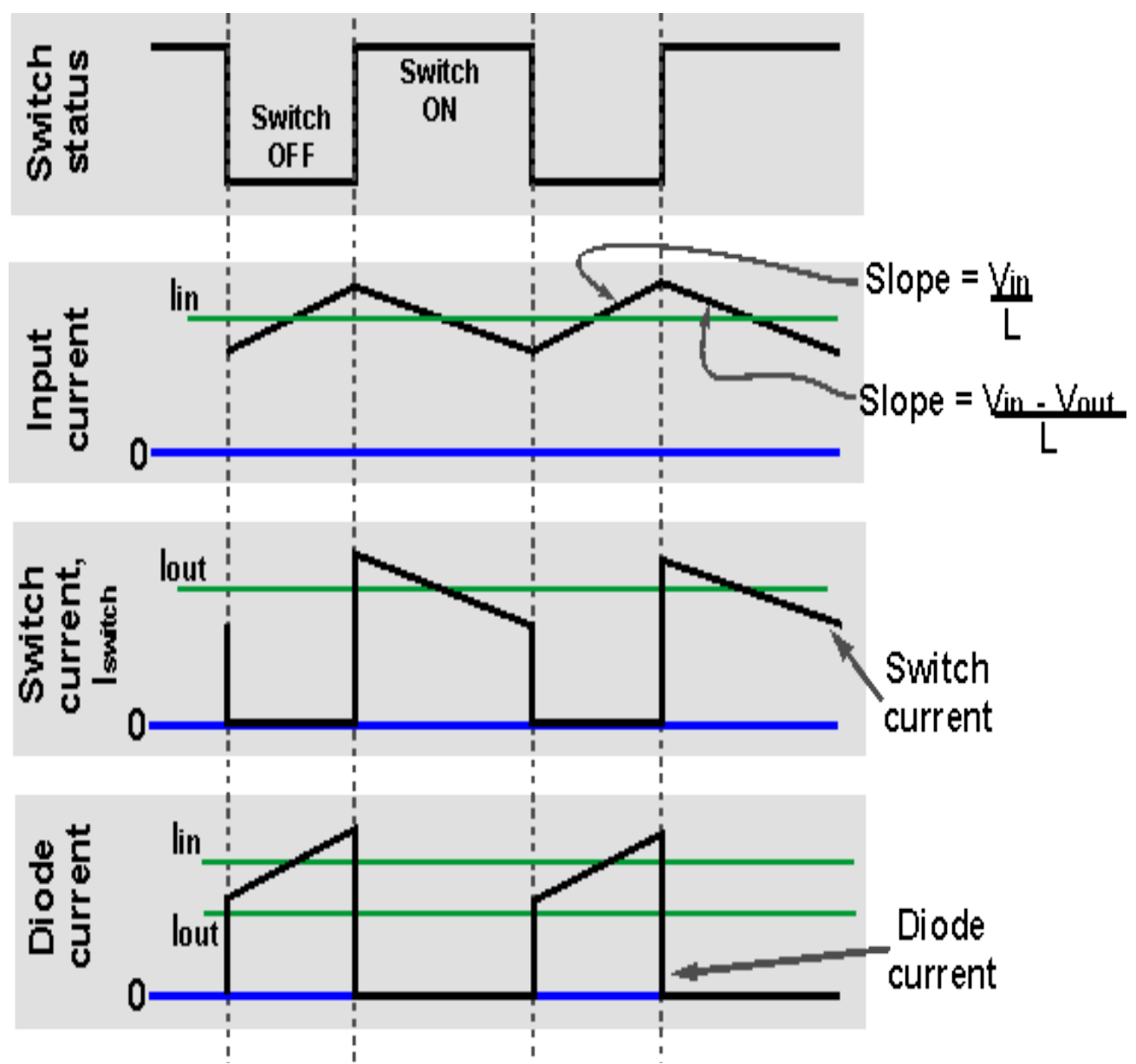

Fig 10: Boost converter 
The output of the project is obtained experimentally as shown below.

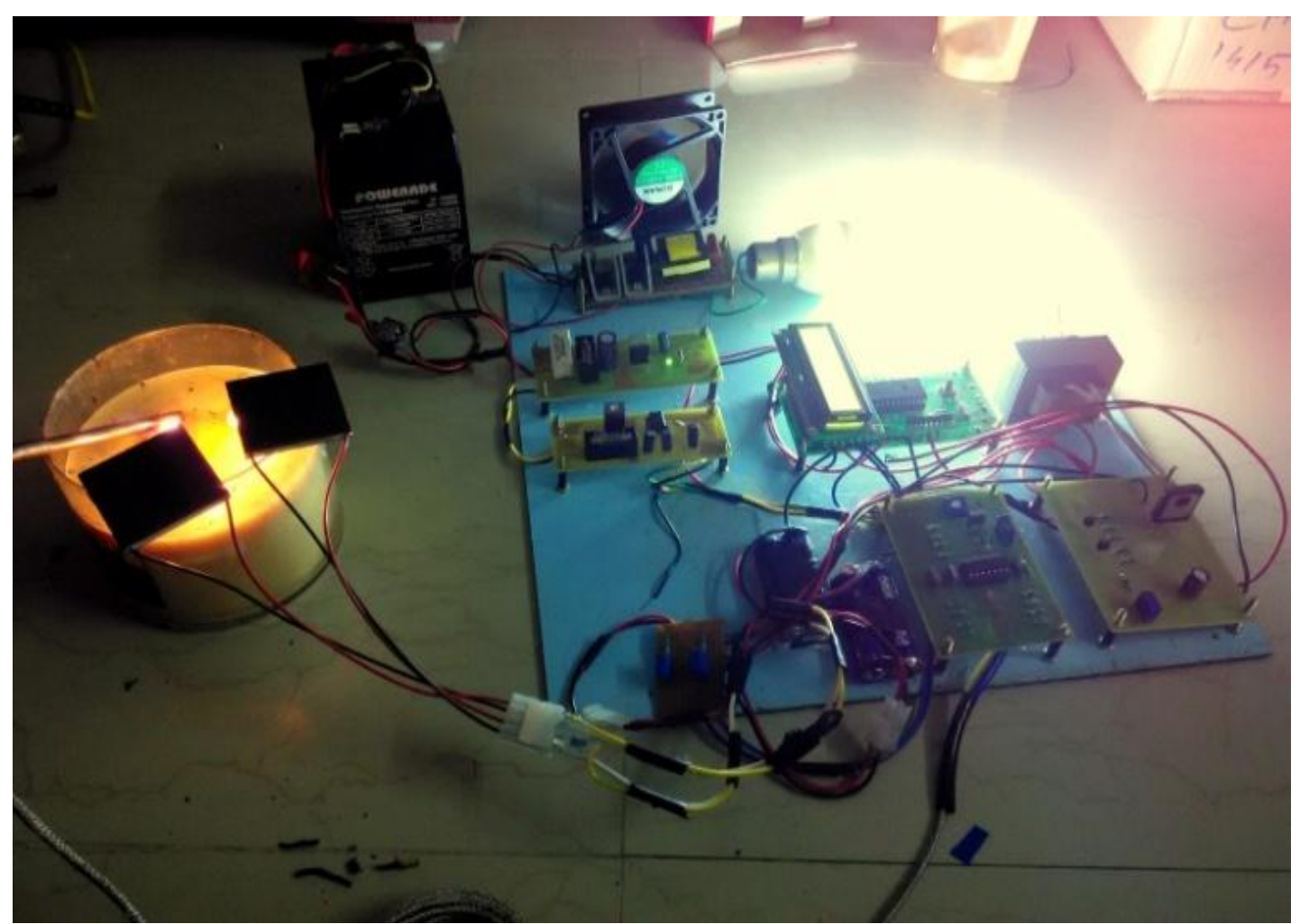

Fig 11: Experimental output

\section{CONCLUSION}

There is an increase in the demand for energy for our day to day activities, from simple devices to complex systems. All these systems depend on the electricity board or the power company for its operation. At one point of time, the scarcity for fuels occurs causing the scarcity in electricity. Hence, it is important to conserve energy. This project aims to conserve the electrical energy to some extent, by trapping the waste heat from the heat source in automobiles. This project can also be applicable in home appliances, where the heat from gas stoves can be trapped for producing electrical energy [2]. By the efficient use of waste heat energy, we can save some amount of energy for operating appliances and auxiliary systems in vehicles.

\section{REFERENCES}

[1] Chang-Hao Piao, Qing Teng, Xing-Yi Wu and Sheng Lu, "Study of Energy Harvesting System Based on Seebeck Effect", International Journal of Advances in Sceince and Technology (IJAST), ISSN 2348-5426, Pages 45-50.

[2] Risha Mal, Rajendra Prasad, Virendra Kumar Vijay, Amit Ranjan, Ratnesh Tiwari, "Thermoelectric Power Generator Integrated Cookstove: A Sustainable Approach of Waste Heat to Energy Conversion", International Journal of Research in Engineering and Technology (IJRET), Volume 3, Special Issue 12, June 2014, Pages 35-40.
[3] AP Rammohan, Manjunath Swamy HM, "Home Made Electricity from Gas stove”, International Journal of Engineering Research and Development (IJERD), Volume 8, Issue 6, September 2013, Pages 29-34.

[4] Omar Abdel-Rahim, Mohamed Orabi, Emad Abdelkarim, Mahrous Ahmed and Mohamed Z. Youssef, “ Switched Inductor Boost Converter for PV applications", Institute of Electrical and Electronics Engineers (IEEE) 2012, 978-1-4577-1216-6, Pages 2100-2106.

[5] Borrnert, T, Burki, T, "Waste heat conversion into electricity", Institute of Electrical and Electronics Engineers, Cement Industry Technical Conference, 2010 IEEE-IAS/PCA 52nd, Pages 1-8.

[6] Dr.R.Seyezhai, V.Aarthi, "Comparison of Interleaved Boost Converter Topologies with Voltage Multiplier for Battery Charging Of PHEV", International Journal of Innovative Research in Computer and Communication Engineering, Vol. 2, Issue 11, November 2014, Pages 6953- 6959.

[7] Hun Sik Han, Yun Ho Kim, Seo Young Kim, Sukkee Um, Jae Min Hyun "Performance Measurement and Analysis of a Thermoelectric Power Generator", Institute of Electrical and Electronics Engineers (IEEE), 2010, 978-1-4244-5343-6/10. 